\title{
The Probable Fate of the Standard Model
}

\author{
José Ramón Espinosa*† \\ ICREA at IFAE \\ Universitat Autònoma de Barcelona \\ 08193 Bellaterra, Barcelona, SPAIN \\ E-mail: espinosa@ifae.es
}

In this talk I describe what we can learn on the fate of the SM (depending on the UV behaviour of the Higgs quartic coupling) from our incomplete knowledge of the Higgs mass, $M_{h}$. I start by reminding you how $M_{h}$ determines that fate and then I use the most up-to-date information we have on $M_{h}$ (both from global EW fits and direct searches at LEP and the Tevatron) to try and extract some conclusions on what that fate might be. At the end I also give you some idea of how future knowledge can improve those conclusions.

European Physical Society Europhysics Conference on High Energy Physics, EPS-HEP 2009, July 16 - 222009

Krakow, Poland

* Speaker.

${ }^{\dagger}$ Work done in collaboration with John Ellis, Gian Giudice, Andreas Hoecker and Toni Riotto, from CERN, see [1]. 

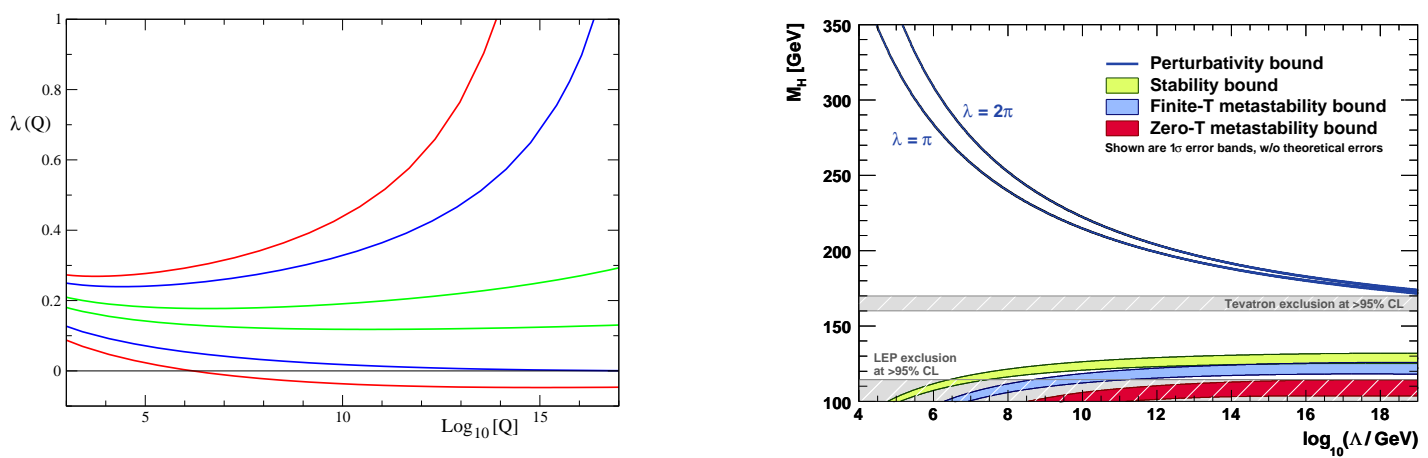

Figure 1: The SM Higgs quartic $\lambda$ running with the renormalization scale $Q$ for several values of $M_{h}$ (left). Perturbativity and (meta)stability bounds on $M_{h}$ vs. the SM cutoff $\Lambda$ (right).

As you know, depending on the Higgs mass, $M_{h}$, the Higgs quartic coupling $\lambda$ can do one of three things when extrapolated up to very high energy (always assuming no new physics beyond the SM), see fig. 1, left panel: it can blow up to nonperturbative values if $M_{h}$ is high enough; it can turn negative at some scale $\Lambda$ (causing a potential instability beyond $\Lambda$ ); or it can stay positive and perturbative all the way up to the Planck mass for intermediate Higgs masses. The blue lines mark the boundaries between these regimes and correspond to the nonperturbative and stability bounds.

I plot such bounds on $M_{h}$ [2] as a function of the SM cutoff in fig. 1, right panel. The upper blue curves show the bound above which lies the nonperturbative regime [chosen here as $\lambda(\Lambda)=2 \pi$. For comparison I also show the $\lambda(\Lambda)=\pi$ line]. The green band gives the lower bound for absolute stability: below it the potential has a very deep nonstandard minimum at high scales that makes the EW one metastable. Such EW vacuum is accepatble as long as its lifetime is long enough. However, below the blue band thermal fluctuations in the early universe can trigger this decay if the temperatures are, roughly speaking, as high as the instability scale (whether this happens or not depends on the cosmological evolution [3]). In any case, below the red band the EW vacuum would have decayed by quantum tunneling with a lifetime shorter than the age of the universe. The widths of these bands are due to the uncertainties in $M_{t}=173.1 \pm 1.3 \mathrm{GeV}$ and $\alpha_{s}=0.1193 \pm 0.0028$ (the theoretical uncertainty, of $1-3 \mathrm{GeV}$ is not shown here, see [1]). These bounds give the most upto-date calculation. The relevance of these bounds is of course that they can offer information on the scale of new physics.

To take full advantage of the previous bounds it is of course crucial to know $M_{h}$, which we don't. By performing a global fit of the SM to EW data, which is logarithmically sensitive to $M_{h}$ through quantum corrections, we can see indirectly what values of $M_{h}$ are preferred. The most upto-date analysis of this kind has been performed by the Gfitter group [4] and was discussed in [5]. Fig. 1 of [5] shows the $\Delta \chi^{2}$ of that EW fit vs. $M_{h}$ including information from direct experimental Higgs searches and showing the impact of the Tevatron [6] around $M_{h}=160-170 \mathrm{GeV}$. Notice that the fit is affected also outside the Tevatron exclusion limit whenever there is sensitivity to the Higgs. The aim of our work was to combine this information on $M_{h}$, the best available today, with the previous bounds, using the full machinery of Gfitter. 


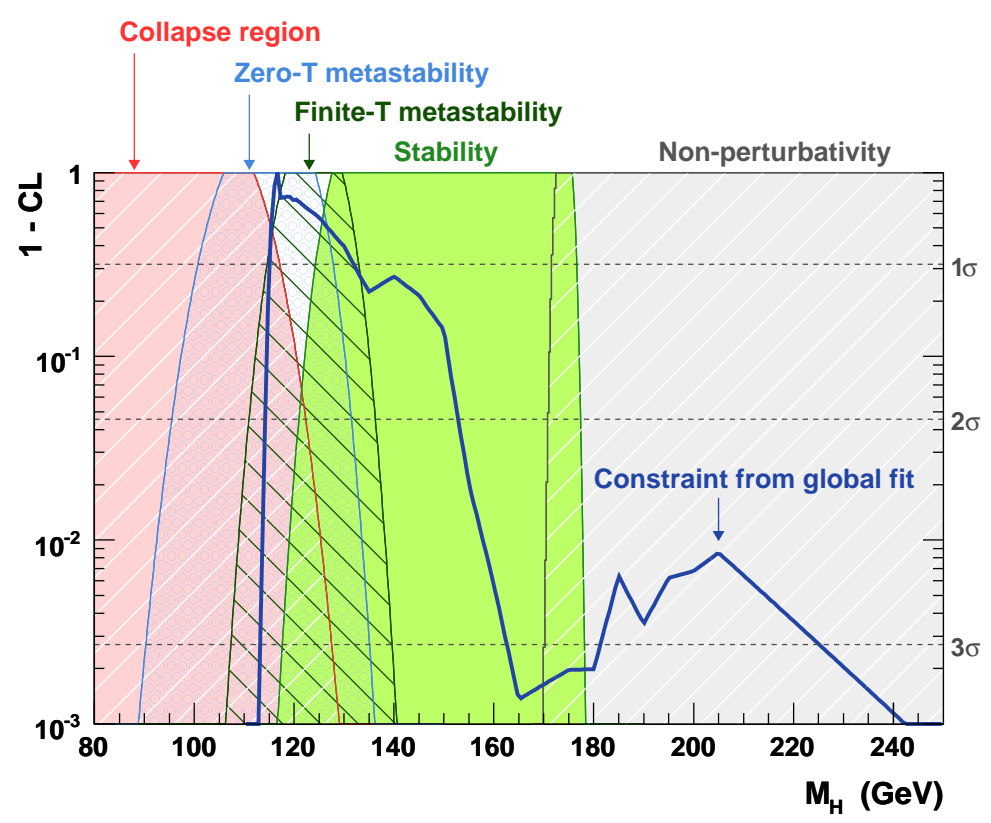

Figure 2: Levels of $1-C L$ vs. $M_{h}$, with different regimes as indicated. Also shown as a blue line is the $1-C L$ function for the combination of current constraints on $M_{h}$.

Fig. 2 shows the kind of results one gets, here for a cutoff $\Lambda=M_{P l}$. The different colored regions correspond to the different regimes of $M_{h}$ discussed before, as indicated. The overlap between regions is due to different uncertainties: the width at the top reflects the theoretical error of the Higgs bounds and the increasing width as one goes down corresponds to the uncertainties in $M_{t}$ and $\alpha_{s}$ mentioned already increasing with $1 \sigma, 2 \sigma$, etc. On top of this we show by a blue line, now as $1-\mathrm{CL}$, the result of the EW fit, including Higgs experimental searches as discussed before. From this we can conclude that the nonperturbative regime is excluded at $99.1 \% \mathrm{CL}$, number greatly influenced by the Tevatron results. The collapse region, with a very unstable EW vacuum, is most affected by the LEP bound [7] but cannot be excluded yet: its $p$-value for compatibility with that bound is 0.4 . The stability region is getting eroded by the Tevatron at its upper end but at the present time one cannot conclude which of the different regimes of (meta)stability is most likely.

For lower values of the cutoff the situation is given by fig. 3. The right plot includes experimental searches while the left doesn't. One can see how the Tevatron wipes out the heavy Higgs region. The most interesting region, though, is that of low mass (actually preferred by the EW fit) because in it one can hope for an upper bound on the cutoff, once $M_{h}$ is measured. So, can we already get some information on the SM cutoff $\Lambda$ ? Not any time soon: even assuming an upper limit of $127 \mathrm{GeV}$ on $M_{h}$, as could be achieved by ATLAS with $1 \mathrm{fb}^{-1}$ of data at $\sqrt{s}=14 \mathrm{TeV}$. As fig. 6, left plot, of [5] shows, one cannot even get a bound on $\Lambda$ at the $1 \sigma$ level. The situation would improve drammatically if a light Higgs is found (with mass roughly below $124 \mathrm{GeV}$ ). For instance, for a Higgs of $115 \mathrm{GeV}$, with a $0.1 \%$ precision in its mass determination, which is achievable at the LHC, one would obtain an upper bound on the cutoff of $\Lambda \sim 10^{8} \mathrm{GeV}$. The bound gets weaker with increasing Higgs mass. 

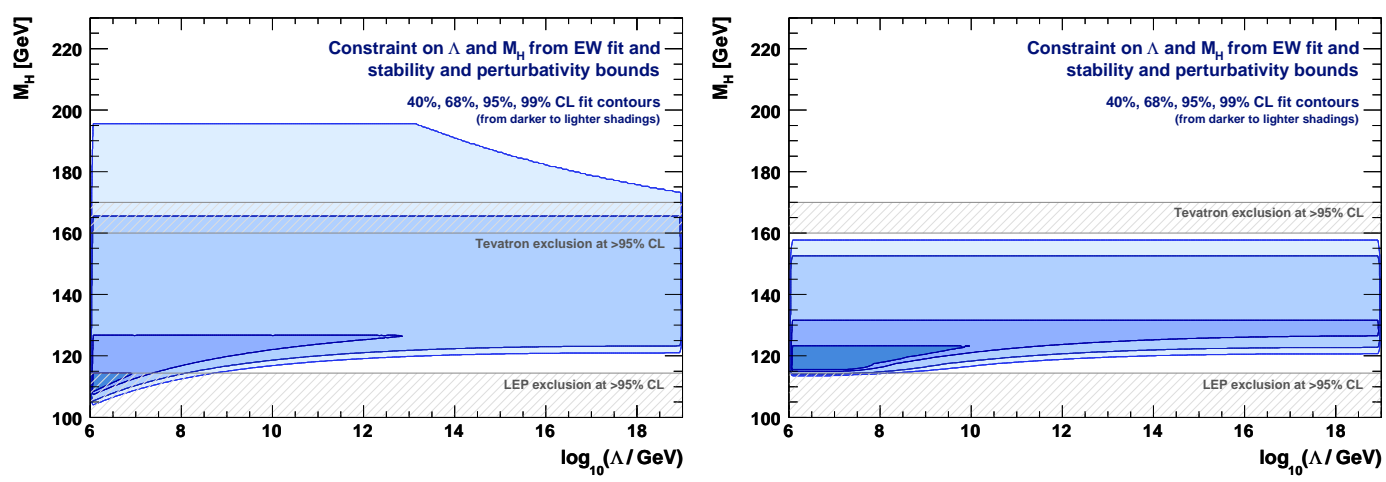

Figure 3: Contours of 40\%, 68\%, 95\% and 99\% CL obtained from scans of fits with fixed values of the variables $M_{h}$ and $\log _{10}(\Lambda / G e V)$. The fits include the $E W$ precision data and the bounds from the perturbativity and stability requirements shown in Fig. 1. The right plot also incorporates the (95\% CL) direct Higgs boson searches at LEP and the Tevatron.

To conclude, it is worth stressing that simply knowing the Higgs mass can be an indication for the existence of new physics much below the Planck mass, as we have just seen. Before we get there we can already use the available information on $M_{h}$ (from EW fits and experimental searches) with some advantage. For instance, we can already exclude the nonperturbative regime of heavy Higgs masses at the $99.1 \% \mathrm{CL}$, while other regimes start to be disfavored. Clearly, stronger conclusions will require more data, which is on its way, so, stay tuned!

\section{References}

[1] J. Ellis, J. R. Espinosa, G. F. Giudice, A. Hoecker and A. Riotto, [hep-ph/0906.0954].

[2] N. Cabibbo, L. Maiani, G. Parisi and R. Petronzio, Nucl. Phys. B 158, 295 (1979); P. Q. Hung, Phys. Rev. Lett. 42, 873 (1979); M. Lindner, Z. Phys. C 31, 295 (1986); M. Lindner, M. Sher and H. W. Zaglauer, Phys. Lett. B 228, 139 (1989); M. Sher, Phys. Rept. 179, 273 (1989); P. B. Arnold, Phys. Rev. D 40 (1989) 613; P. Arnold and S. Vokos, Phys. Rev. D 44 (1991) 3620; B. Schrempp and M. Wimmer, Prog. Part. Nucl. Phys. 37, 1 (1996); M. Sher, Phys. Lett. B 317, 159 (1993); G. Altarelli and G. Isidori, Phys. Lett. B 337, 141 (1994); G. Isidori, G. Ridolfi and A. Strumia, Nucl. Phys. B 609, 387 (2001), [hep-ph/0104016]; J. A. Casas, J. R. Espinosa and M. Quirós, Phys. Lett. B 342, 171 (1995), [hep-ph/9409458]; Phys. Lett. B 382, 374 (1996), [hep-ph/9603227]; J. R. Espinosa and M. Quirós, Phys. Lett. B 353 (1995) 257 [hep-ph/9504241]; T. Hambye and K. Riesselmann, Phys. Rev. D 55, 7255 (1997), [hep-ph/9610272].; C. F. Kolda and H. Murayama, JHEP 0007, 035 (2000), [hep-ph/0003170].

[3] J. R. Espinosa, G. F. Giudice and A. Riotto, JCAP 0805, 002 (2008), [hep-ph/0710.2484].

[4] H. Flächer et al., Eur. Phys. J. C 60, 543 (2009) [hep-ph/0811.0009]; updated results taken from http://cern.ch/gfitter.

[5] A. Hoecker, these Conference Proceedings.

[6] Tevatron New Phenomena and Higgs Working Group for the CDF and D0 Collaborations, FERMILAB-PUB-09-060-E, [hep-ex/0903.4001].

[7] The ALEPH, DELPHI, L3 and OPAL Collaborations, and LEP Working Group for Higgs Boson Searches, Phys. Lett. B 565, 61 (2003), [hep-ex/0306033]. 